Journal of Computational Neuroscience 3, 91-110 (1996)

(C) 1996 Kluwer Academic Publishers. Manufactured in The Netherlands.

\title{
Inhibition Synchronizes Sparsely Connected Cortical Neurons Within and Between Columns in Realistic Network Models
}

\author{
PAUL BUSH* \\ Howard Hughes Medical Institute, Computational Neurobiology Laboratory, Salk Institute, La Jolla, \\ CA 92037, USA \\ TERRENCE SEJNOWSKI \\ Departments of Biology and Neuroscience, University of California at San Diego, La Jolla, CA 92093, USA
}

Received July 5, 1994; Revised September 7, 1995; Accepted October 10, 1995

Action Editor: Rodney Douglas

\begin{abstract}
Networks of compartmental model neurons were used to investigate the biophysical basis of the synchronization observed between sparsely-connected neurons in neocortex. A model of a single column in layer 5 consisted of 100 model neurons: 80 pyramidal and 20 inhibitory. The pyramidal cells had conductances that caused intrinsic repetitive bursting at different frequencies when driven with the same input. When connected randomly with a connection density of $10 \%$, a single model column displayed synchronous oscillatory action potentials in response to stationary, uncorrelated Poisson spike-train inputs. Synchrony required a high ratio of inhibitory to excitatory synaptic strength; the optimal ratio was $4: 1$, within the range observed in cortex. The synchrony was insensitive to variation in amplitudes of postsynaptic potentials and synaptic delay times, even when the mean synaptic delay times were varied over the range 1 to $7 \mathrm{~ms}$. Synchrony was found to be sensitive to the strength of reciprocal inhibition between the inhibitory neurons in one column: Too weak or too strong reciprocal inhibition degraded intra-columnar synchrony. The only parameter that affected the oscillation frequency of the network was the strength of the external driving input which could shift the frequency between 35 to $60 \mathrm{~Hz}$. The same results were obtained using a model column of 1000 neurons with a connection density of $5 \%$, except that the oscillation became more regular.

Synchronization between cortical columns was studied in a model consisting of two columns with 100 model neurons each. When connections were made with a density of $3 \%$ between the pyramidal cells of each column there was no inter-columnar synchrony and in some cases the columns oscillated $180^{\circ}$ out of phase with each other. Only when connections from the pyramidal cells in each column to the inhibitory cells in the other column were added was synchrony between the columns observed. This synchrony was established within one or two cycles of the oscillation and there was on average less than $1 \mathrm{~ms}$ phase difference between the two columns. Unlike the intra-columnar synchronization, the inter-columnar synchronization was found to be sensitive to the synaptic delay: A mean delay of greater than $5 \mathrm{~ms}$ virtually abolished synchronization between columns.
\end{abstract}

Keywords: synchronized oscillations, computer modeling, cortical microcircuitry

\section{Introduction}

${ }^{*}$ Current Address: Keck Center for Integrative Neuroscience, University of California, San Francisco, 513 Parnassus Avenue, Box 0444, San Francisco, CA $94143-0444$.
Although the traditional role for inhibition has been to regulate the level of excitation, recent experimental 
and modeling studies suggest an additional function for inhibition in cortex: Regulating the timing of action potential occurrence (Bush and Sejnowski, 1994; Gray et al., 1992; Lytton and Sejnowski, 1991). In this study, we explore the influence of inhibition on the temporal pattern of spike firing both within and between columns consisting of small networks of sparsely connected model neurons.

Synchronous oscillatory firing of populations of cortical neurons at frequencies around $40 \mathrm{~Hz}$ has been observed within and between many different cortical areas in primates and cats both awake and anaesthetized (for recent review see Singer (1993)). It has been proposed that this synchronous activity is used to group separated parts of single objects (Engel et al., 1991a; Sporns et al., 1991) and even signal visual awareness (Crick and Koch, 1990). Although there is still much debate as to the role and significance of synchrony, it is generally agreed that many cortical neurons can fire synchronously under some conditions. Therefore, it is worthwhile to discover exactly how cortical tissue generates and sustains synchronized oscillatory firing of its component neurons, both within a single column and between columns that may be located in different hemispheres (Engel et al., 1991b). For simplicity, we have restricted our model to a single layer of cortex (layer 5). Slice experiments have shown that isolated layer 5 is capable of intrinsic generation of oscillatory activity, although at lower frequencies than those discussed here (Silva et al., 1991). It is possible that intrinsic bursting cells in vivo have significantly higher bursting frequencies than those in vitro, and it is also possible that there are other populations of intrinsic bursting cells with different intrinsic frequencies (McCormick et al., 1993).

A number of other studies have modeled synchronized oscillations in cortex at a simpler level of physiological realism, many of them focusing on how such activity might be useful, for example in perceptual grouping (Koenig and Schillen, 1991; Sporns et al., 1989). This study does not address the function of synchronization; instead we have constructed a physiologically realistic network model based on ionic currents and conductances, with every parameter directly representing a physiological variable. We intended to determine whether the presently available data on the physiology and microanatomy of cortex are sufficient to explain how synchronized oscillatory firing occurs and how certain physiological variables affect this activity. We found both intra- and inter-columnar synchronization to be robust to changes in parameters known to have a wide degree of variation in cortex. Some parameters did affect synchronization, such as the degree of connectivity and the involvement and interaction of inhibitory interneurons.

A previous pilot study (Bush and Douglas, 1991), using a simplified biophysical model of a cortical column, established a basic mechanism for cortical synchronization to arise. This model included a number of simplifications: It consisted of only 11 fully connected cells and each synapse had to be spread out in time as well as increased in amplitude to compensate. One of the simplifications led to an artifactual phenomenon explained in the Discussion. The present model is a more faithful simulation of a piece of cortical tissue, incorporating a more realistic model reduced cell, many more cells, sparse connectivity and connections between as well as within columns. This more accurate model not only produced results that were better fits to the experimental data, but also allowed us to determine systematically how and in what way each physiological variable affected synchronization, which was not possible with the simpler model.

We have also modeled the interactions that occur between columns. When long-range horizontal connections between columns were first discovered in neocortex they seemed to imply a paradox: These connections extended far beyond the receptive fields of single cells, yet stimulating the surrounding area outside the receptive fields of undriven cells was not effective in driving them (Gilbert et al., 1990). This suggested that perhaps these connections were relatively weak, and indeed the density of synaptic connectivity is much higher within a column than between columns (Kisvarday and Eysel, 1992; Martin, 1988). Although excitatory postsynaptic potentials (EPSPs) can be generated in neurons when long range horizontal afferents are stimulated, it is not possible to generate action potentials by stimulating these fibers alone (Hirsch and Gilbert, 1991). 'This suggests that these connections might have more of a modulatory role. We have used our model to simulate inter-columnar connectivity to explore this possibility.

\section{Methods}

\section{Model Neurons}

Simulations were performed using standard techniques for compartmental models of branching dendritic trees (Rall, 1964). The primary neuron used in our networks 
was a layer 5 pyramidal neuron modeled by $9 \mathrm{com}$ partments. This model neuron was reduced from a 400 compartment model of a reconstructed cat visual cortex pyramidal cell (Koch et al., 1990) using a method that preserves essential electrotonic parameters (Bush and Sejnowski, 1993). The reduced model pyramidal cell had a specific membrane resistance $R_{m}=7042 \Omega \mathrm{cm}^{2}$ and a specific capacitance $C_{m}=2.84 \mu \mathrm{F} / \mathrm{cm}^{2}$ which produced an input resistance $R_{\mathrm{in}}=45 \mathrm{M} \Omega$ and a membrane time constant $\tau_{m}=20 \mathrm{~ms}$. Since approximately $20 \%$ of neocortical cells are inhibitory (Douglas and Martin, 1990) $20 \%$ of our cells were 7 compartment inhibitory (basket) neurons (not based on a reconstructed cell) with $C_{m}=2.21 \mu \mathrm{F} / \mathrm{cm}^{2}$ and $R_{m}=6800 \Omega \mathrm{cm}^{2}$ giving an $R_{\mathrm{in}}=164 \mathrm{M} \Omega$ and $\tau_{m}=15 \mathrm{~ms}$. The values of $C_{m}$ and $R_{m}$ for the reduced cells are respectively larger and smaller than for the full cells to compensate for the reduction in surface area (Bush and Sejnowski, 1993). For all model cells the axial resistivity was $200 \Omega \mathrm{cm}$ and the resting membrane potential was $-55 \mathrm{mV}$ ('resting' assumes background synaptic activity producing a steady depolarization). The values chosen for these passive parameters are within the typical range observed in neocortical cells in vivo and are have been discussed elsewhere (Bush and Sejnowski, 1994).

Each model neuron had Hodgkin-Huxley-type active conductances at the soma only. These conductances were implemented exactly as described in Bush and Sejnowski (1994), using a kinetic scheme developed by Borg-Graham (1987). The pyramidal cells had fast sodium and potassium conductances $(\mathrm{gNa}+\mathrm{gKd})$ to produce action potentials, a fast high-threshold calcium conductance ( $\mathrm{gCa}$ ) to introduce calcium into the cell during each spike, and a calcium-dependent potassium conductance ( $\mathrm{gKca}$ ) to produce hyperpolarizations that terminated bursts of spikes. Intracellular calcium accumulated in the soma compartment and decayed exponentially to its resting value with a time constant that was different for each cell, between $10-50 \mathrm{~ms}$. The different calcium decay rates gave each pyramidal cell a different intrinsic bursting frequency. The basket cells had only fast sodium and potassium conductances and fired continuous trains of high-frequency spikes to constant current input. Figure 1 shows the intrinsic firing properties of the model pyramidal and baskets cells.

During network simulations, noise was injected into the soma of every model neuron in the form of a current that changed every time step to a random number uniformly distributed between positive and negative $1 \mathrm{nA}$ for pyramidal cells and $0.3 \mathrm{nA}$ for basket cells. This method produced a varying resting membrane potential similar to that seen in vivo due to background synaptic inputs, without changing $R_{\text {in }}$ and $\tau_{m}$. This background noise made synchronization more difficult and produced a background resting firing rate of a few $\mathrm{Hz}$.

\section{Synaptic Connectivity}

Synaptic conductances were modeled using an alpha function conductance change ( $1 \mathrm{~ms}$ to peak) activated on the postsynaptic dendrite with some delay after the presynaptic spike (Bernander el al., 1991). Peak amplitudes and synaptic delays for each connection were randomly assigned according to a Gaussian distribution with a standard deviation equal to half the mean. This reproduced the large range of delays and postsynaptic potential (PSP) amplitudes observed experimentally. The mean synaptic delay time was $1.2 \mathrm{~ms}$ with a fixed minimum of $0.5 \mathrm{~ms}$ (Mason et al., 1991; Nicoll and Blakemore, 1990). Mean peak postsynaptic conductance amplitudes were varied but were typically 1-2 nS except for inhibitory synapses on pyramidal cells, which were typically $4-8 \mathrm{nS}$. This reflects the observations of Komatsu et al. (1988) who found that single inhibitory conductance changes were significantly larger than single excitatory ones. This may reflect the fact that basket cells typically make multiple synaptic contacts on the proximal dendrites of a target pyramidal ccll whilc pyramidal cclls only make one or a few contacts per target (Somogyi et al., 1983; Gabbot et al., 1987). The reversal potential for EPSPs was $0 \mathrm{mV}$ and that for inhibitory postsynaptic potentials (IPSPs) was $-65 \mathrm{mV}(10 \mathrm{mV}$ below the resting potential) (Connors et al., 1988).

Excitatory synapses on model pyramidal cells were made on the terminal basal and oblique dendritic compartments, whereas inhibitory synapses were made onto the soma and proximal dendrites. Excitatory and inhibitory synapses were made onto all compartments of the basket cells (Douglas and Martin, 1990). Pyramidal cells received external driving input from uncorrelated Poisson spike trains. Typically each cell received four $20 \mathrm{nS}$ excitatory synapses active at a mean rate of $200 \mathrm{~Hz}$. This is equivalent to a larger number of inputs active at a lower rate.

Connectivity within a single column, at the scale of 100-200 $\mu \mathrm{m}$, appears to be random in the sense that axons make contacts on all potential targets within their zone of arborization (White, 1989). Thus, there was no spatial topography within our model columns: Every 


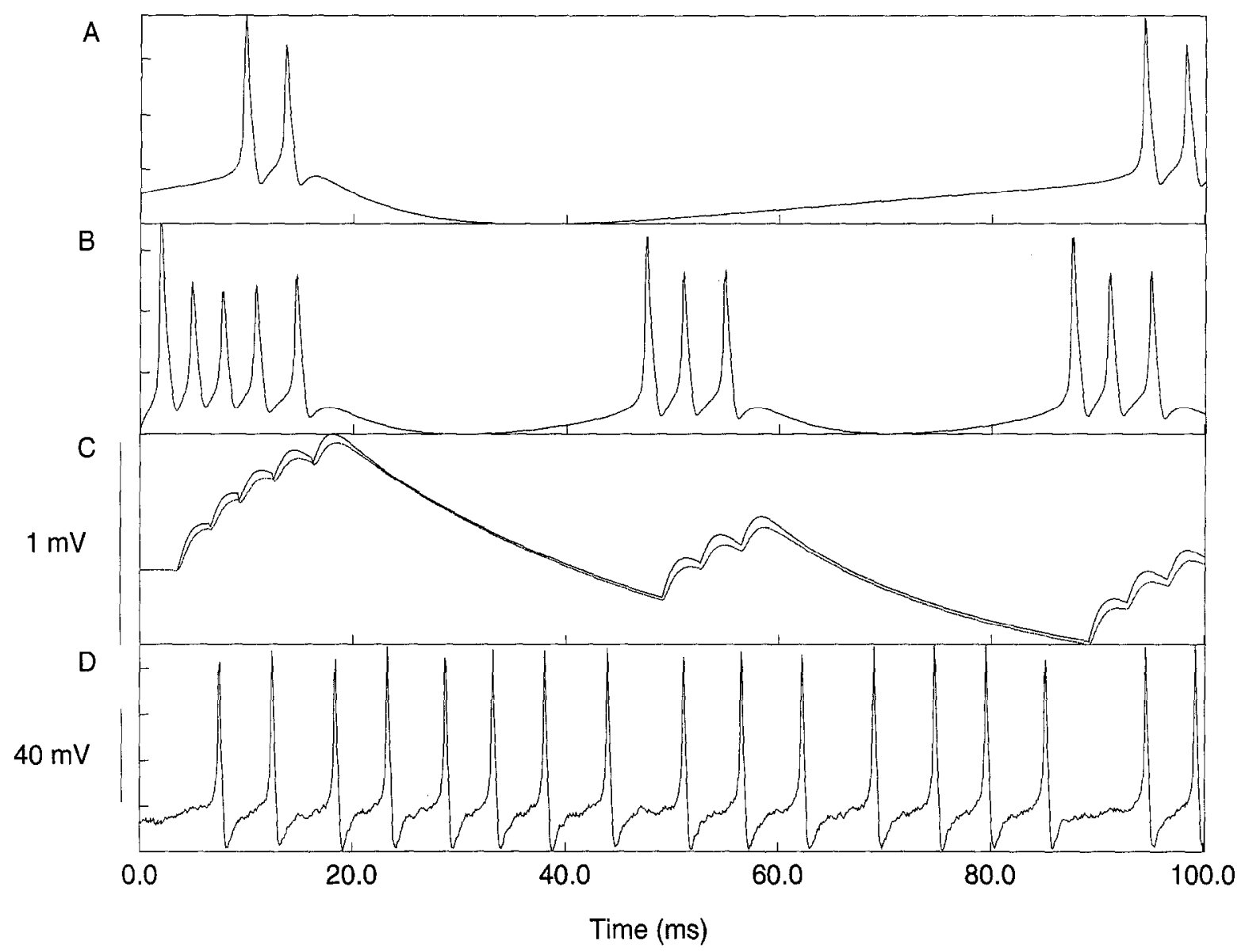

Figure 1. Intrinsic firing properties of isolated model neurons. A) Bursting response of a pyramidal cell when injected with constant $0.2 \mathrm{nA}$ depolarizing current at the soma. B) Higher frequency response to a $1 \mathrm{nA}$ current. The stronger input also produces more spikes per burst C) Postsynaptic response of a second pyramidal cell connected with a $0.5 \mathrm{nS}$ synapse to the cell shown in (A). Top trace is the dendritic potential, bottom trace is the somatic potential. D) Spike train from a basket cell injected with $0.05 \mathrm{nA}$ depolarizing current and $+/-0.3 \mathrm{nA}$ noise (see Methods).

cell had an equal chance of contacting every other cell (but no self-connections were allowed). The density of connectivity within a column has been estimated by dual intracellular impalement and spike-triggered averaging to be $5-15 \%$ (Komatsu et al., 1988; Mason et al., 1991; Thomson et al., 1988), although it may be lower in the deeper layers (Nicoll and Blakemore, 1990, 1993; Thomson et al., 1993). We explored a range of different connection densities: For the 100 neuron columns presented here we used a connectivity of $10 \%$. Thus each model cell received input from exactly 8 pyramidal cells and 2 baskets cells, randomly chosen. Since each neuron received input from only 10 other neurons in the column, it was necessary to add a scaling factor to the peak synaptic strengths. For a 100 neuron column with $10 \%$ connectivity all synaptic strengths were multiplied by 10 . For a 1000 neuron column with $10 \%$ connectivity no scaling factor was necessary.

In order to view rapidly and easily the average activity of the whole network, an analog of the local field potential (LFP) was calculated for each simulation, called the local averaged potential (LAP). This was a running average of all the membranc potentials of all the pyramidal cell somas. The presence of oscillations in this LFP analog was an indicator of synchronized activity in the pyramidal cell population. Our LAP is not directly equivalent to a real LFP, which is a sum of all the local currents, both voltage- and ligand-gated, weighted by the distance of the sources from the electrode. However, our LAP is a direct measure of the average neuronal activity and for the task of showing 
synchronous oscillations it is actually better suited than the real LFP.

A quantitative measure of the degree of synchronization between two columns is provided by the correlation amplitude (CA), ranging from -1 to +1 , which is the height of the peak closest to zero in the crosscorrelation of the two network LAPs (Gray et al., 1992). The phase shift of this peak from zero provides a measure of the tightness of the synchronization. For single columns the CA is measured for the first peak away from zero in the auto-correlation of the LAP. This number reflects the regularity and amplitude of the oscillatory discharges of the synchronized column. The phase shift of this peak gives the period of the oscillation.

For some data the power and phase spectra were calculated using the software package ACF/gr (Paul Turner, Oregon Graduate Institute of Science and Technology) which calculates the spectrum by

$$
\operatorname{Power}(\omega)=\sqrt{x^{2}(\omega)+y^{2}(\omega)}
$$

where $x(w)$ and $y(w)$ are the real and imaginary frequency coefficients computed by the FFT. The phase was calculated by

$$
\operatorname{Phase}(\omega)=\tan ^{-1}\left(\frac{y(\omega)}{x(\omega)}\right)
$$

\section{Simulations}

All simulations were carried out on a MIPS Magnum 3000/33 using a modified version of CABLE (Hines, 1989). We used a time step of $0.1 \mathrm{~ms}$ with 2 nd order correct numerical integration. A simulation with 100 neurons and $10 \%$ connectivity took 9 minutes of computer time to simulate $500 \mathrm{~ms}$ of real time.

\section{Results}

\section{Synchronization within a Single Column}

Figure 2 shows a simulation of a 100 neuron column ( 80 pyramidal cells, 20 basket cells) without synaptic connections. The pyramidal cells were all driven hy uncorrelated Poisson spike train inputs at a mean frequency of $200 \mathrm{~Hz}$, but they had different intrinsic bursting frequencies because of different internal calcium elimination rates (see Methods). The bursting was less regular than in Fig. 1 due to the injection of noise into each pyramidal cell soma. The basket cells received no driving input and only fired a few spontaneous spikes due to background noise. Because there were no synaptic connections, there were no correlations between the neurons and the LAP was flat after the initial transient burst.

Figure 3 shows the output of the same network when the neurons were randomly connected at a density of $10 \%$. Oscillations were visible in the LAP indicating synchronized firing of the pyramidal cells $(\mathrm{CA}=0.56$ and the period of oscillation was $22.2 \mathrm{~ms}$, giving a frequency of $45 \mathrm{~Hz}$ ). The basket cells showed evidence for bursting at the same frequency as the pyramidal cells even thought they have no intrinsic oscillatory properties; they are directly driven by the pyramidal cells, so the fact that they fired in bursts is further evidence of synchronized population discharge. The mechanism of synchronization is detailed in the Discussion.

As observed in experimental recordings in vivo (Gray et al., 1992), the synchronization sometimes spontaneously ceased (in this case at 100 and $350 \mathrm{~ms}$ ) and then reappeared. The basket cell bursts in the model became less clumped at these times. Since the driving input to the network was stationary throughout, these changes were not due to changes in the nature of the external input. The synchronization was a statistical phenomenon, "as observed in the experimental recordings; it was often difficult to see a regular oscillation in the spike train of a single cell, especially if it did not fire many spikes, and of course it was impossible to see synchronization. When examining all 7 single-cell traces, one observes moments when the spikes all lined up, but even in these cases there was significant jitter in individual spike times. Comparing two traces spike by spike, such as the top two pyramidal cell traces in Fig. 3, we often found few instances of simultaneous firing, although on average both cells were locked to the underlying oscillation of the population (shown by the LAP). In other cases (such as the 3rd and 4th trace) the synchronization of spike firing was clearer. It is important to have an averaged measurc of the activity of the whole population (such as the LAP shown here) to determine if the component cells are collectively oscillating in synchrony.

In networks with 100 neurons we could not obtain good synchrony with connection densities of less than $10 \%$, but lower connection densities were effective when simulating networks with more neurons. In Fig. 4, 1000 neurons were connected at a density of $5 \%$, with a synaptic strength scaling factor of 2 (see 


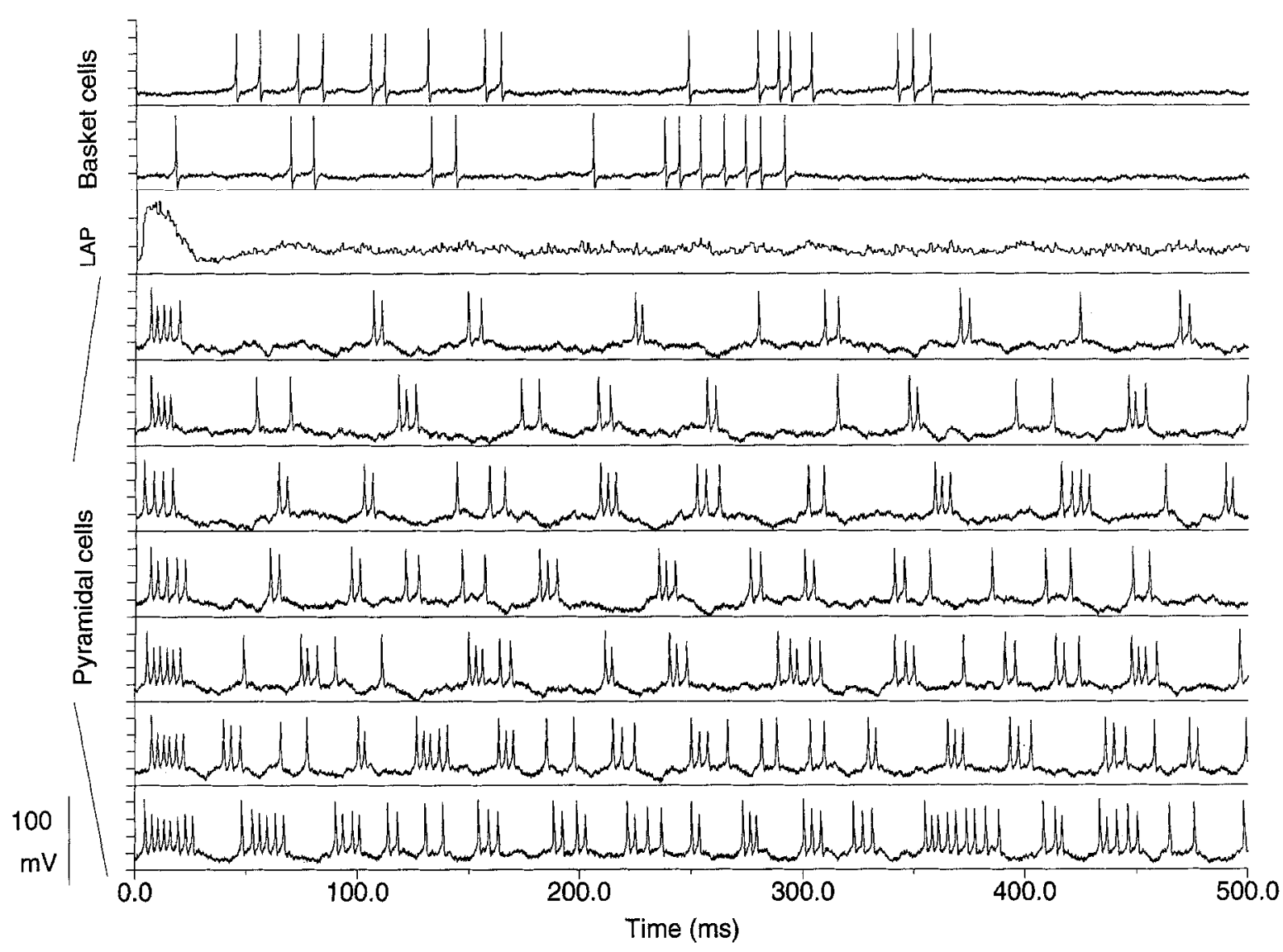

Figure 2. Activity in models of 100 isolated layer 5 cortical neurons. The top 2 traces show the somatic membrane potential of 2 basket cells from a population of 20 making up one column. Since the driving input to the network goes only to the pyramidal cells the basket cells do not fire many spikes. The next trace is an analog of the local field potential, called the local averaged potential (LAP), to show the global synchronization of the whole column (see Methods). The flat trace shown here indicates that the neurons were not firing synchronously. The bottom 7 traces show the somatic membrane potential of 7 sample pyramidal cells from the total population of 80 . In this example the intrinsic bursting frequency of the cells increases towards the bottom.

Methods). We obtained the same results with a connectivity of $10 \%$ and no scaling factor; i.e., the synapses had same strengths as in real cortex. The oscillation and synchronization in a network of 1000 neurons was highly regular, as a consequence of the law of large numbers, although there was still a significant amount of jitter in spike times at the level of single cell traces $(\mathrm{CA}=0.58$ and the period was $19.8 \mathrm{~ms}$, giving a frequency of $50.5 \mathrm{~Hz}$, slightly higher than the 100 cell network). Even though in terms of numbers this simulation may be more realistic than the 100 neuron simulations, the output of the smaller network appears more realistic (less regular). This is considered further in the Discussion.

The membrane potential trace of the 4 th pyramidal cell in Fig. 4 is shown at a higher temporal resolution in Fig. 5. The large compound EPSP present on each cycle of the population oscillation is clearly visible. Spikes only arise from the top of these compound EPSPs, but not every compound EPSP causes a spike. This figure can be compared to Fig. 6, an intracellular recording from a cat visual cortex neuron firing oscillatory bursts during optimal stimulation. There is a rhythmic series of large compound EPSPs, some of which cause spiking. There are no spikes at other times in the phase of the oscillation.

\section{Sensitivity to Parameters}

The distributions of synaptic conductances and time delays in all of our simulations had large standard 


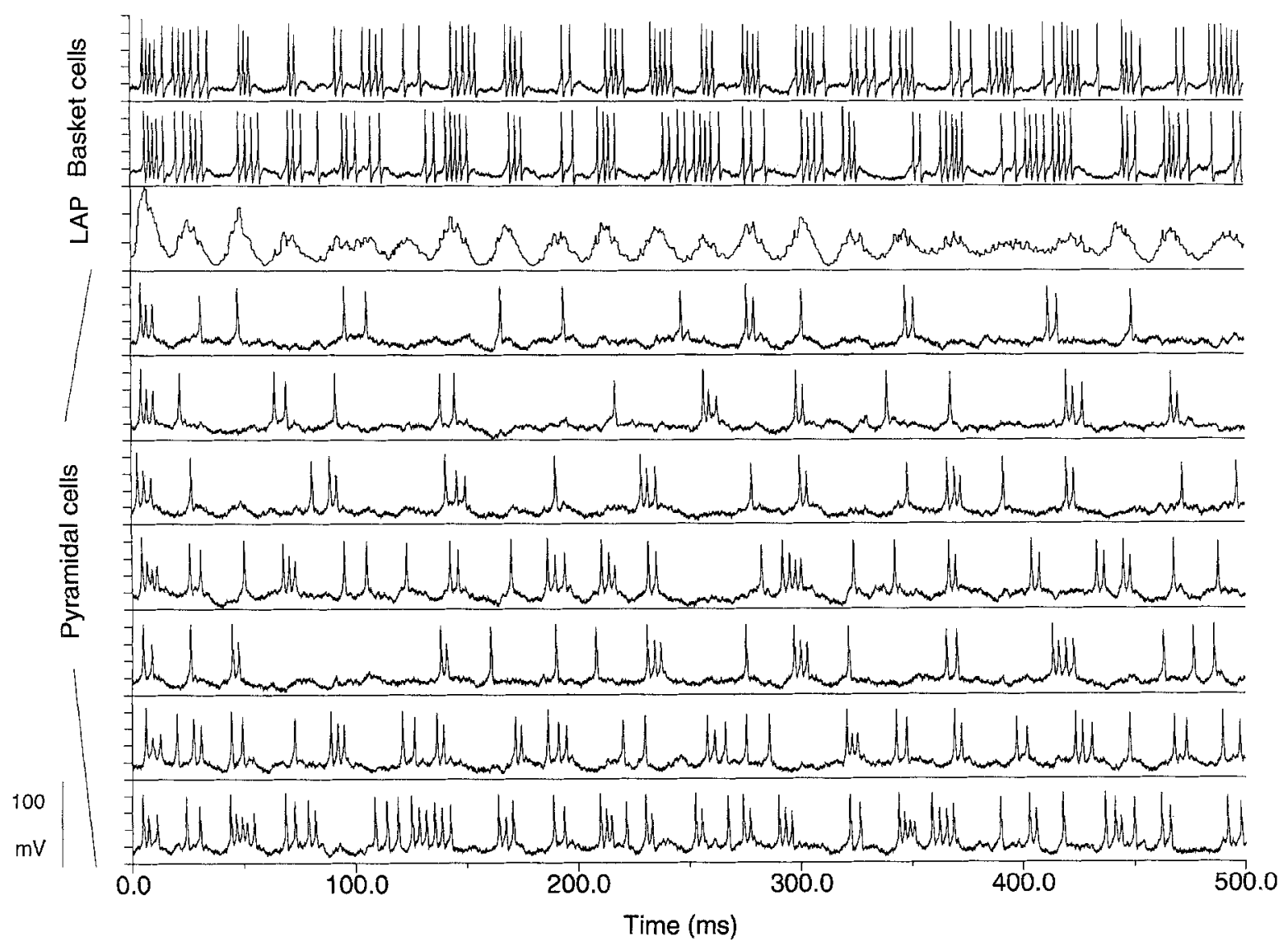

Figure 3. Intracolumnar synchronization in a model network of neurons connected with a probability of $10 \%$. The oscillations in the LAP shown here indicate that the neurons in the column fired synchronously. This is confirmed by the firing of the basket cells, which have no intrinsic bursting dynamics and only fire bursts in response to synchronized input from the pyramidal cells. There is considerable 'jitter' in an individual pyramidal cell's output; the network oscillation is a statistical property of the population and not at all 'clock-like'. The synchrony spontaneously disappears and reappears at 100 and $350 \mathrm{~ms}$. This rapid shift has been seen in real experimental data.

deviations, so that synaptic connections with an overall mean of $1 \mathrm{nS}$ often had values as small as $0 \mathrm{nS}$ and as large as $3 \mathrm{nS}$ and time delays with a mean of $1.2 \mathrm{~ms}$ had values from $0.5 \mathrm{~ms}$ up to as high as $4 \mathrm{~ms}$. The synchronized firing of the network was not affected by this variability, and was in general a robust phenomenon: We repeated all of our simulations with a noise level 4 times higher than that shown here (peak current $4 \mathrm{nA}$ for pyramids, $1.2 \mathrm{nA}$ for baskct cclls--sce Methods) and with a resting membrane potential of $-65 \mathrm{mV}$ instead of $-55 \mathrm{mV}$, but synchronized oscillation was still present. With some sets of parameters (such as higher connectivity, stronger inhibitory synapses and lower resting membrane potentials) we were able to obtain even stronger synchronization, but the values of parameters used for the simulations presented in this paper are probably closer to those in the real cortex.
We found that increasing the mean synaptic delay within a column did not disrupt synchronization, even when the mean delay was as large as $7.2 \mathrm{~ms}$ rather than $1.2 \mathrm{~ms}$ (not shown). In this case, the oscillation became more regular, with every cell firing on every cycle and the cells with the highest intrinsic bursting frequency always leading the others. The frequency of oscillation of the network was also decreased due to the long duration of each burst. The only other way we found to alter the frequency of oscillation of the network was to vary the strength of the external driving input. By changing this parameter it was possible to vary the oscillation frequency of the network in the range $35-60 \mathrm{~Hz}$.

Network synchronization was generally resistant to variations in synaptic strengths. However, inhibitory synapses had to be stronger than excitatory ones. We 


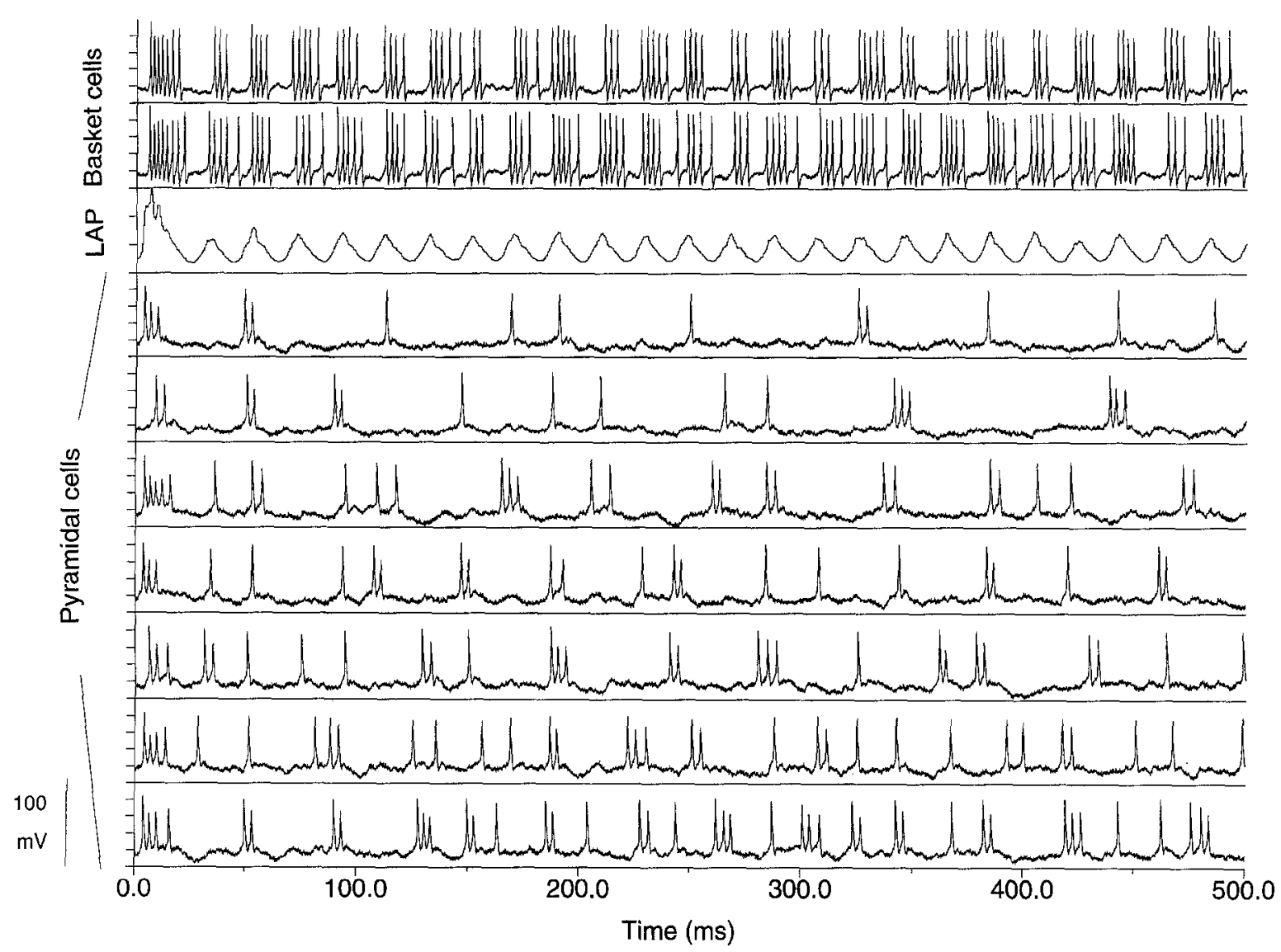

Figure 4. Synchronization of a 1000 neuron network connected at a density of 5\%. The oscillation in this network, apparent in the LAP and rhythmic bursting of the basket cells, is much more regular than that of the 100 neuron network shown in Fig. 3, although there is still significant 'jitter' in the individual pyramidal spike trains.

found that a ratio of approximately $4: 1$, the value initially chosen on the basis of physiological results (see Methods), was optimal for realistic synchronization. In networks without inhibition, simulating the effect of bicuculine, excitatory feedback operates unconstrained producing large paroxysmal burst discharges, with the membrane potentials of the highest intrinsic frequency cells latching up past spike threshold (not shown). The only inhibitory/hyperpolarizing force in these networks was the intrinsic potassium conductance of the pyramidal cells, which was not strong enough to control the excitatory feedback.

The network was also somewhat sensitive to the strength of reciprocal inhibition between the basket cells. Although it is known from anatomical studies that local inhibitory interneurons make synaptic connections on each other (Douglas and Martin, 1990), very little is known about the function of these connections beyond the vague concept of 'disinhibition'. Figure 7B shows the same network LAP as in Fig. $3(\mathrm{CA}=0.56)$ and one of the basket cell traces. Figure 7A is the LAP from the same network with inhibitory contacts between hasket cells removed. Synchrony was slightly weaker $(\mathrm{CA}=0.50)$ because the basket cell bursts were no longer terminated by inhibitory feedback and as a result became less discrete, sometimes continuing into the next cycle. This produced a lower frequency of oscillation ( $38 \mathrm{~Hz}$ compared to $45 \mathrm{~Hz}$ in Fig. 7B). This higher level of inhibitory activity also resulted in less activity in the pyramidal cell population (not shown). When synapses between baskets cells were made very strong (Fig. 7C) synchronization was severely disrupted $(\mathrm{CA}=0.04)$. In this case basket cells were inhibited before they could provide effective inhibition to the pyramidal cell population so the pyramidal cells began to fire continuously instead of in synchronous bursts. Thus, mutual inhibition between inhibitory interneurons within a single column 


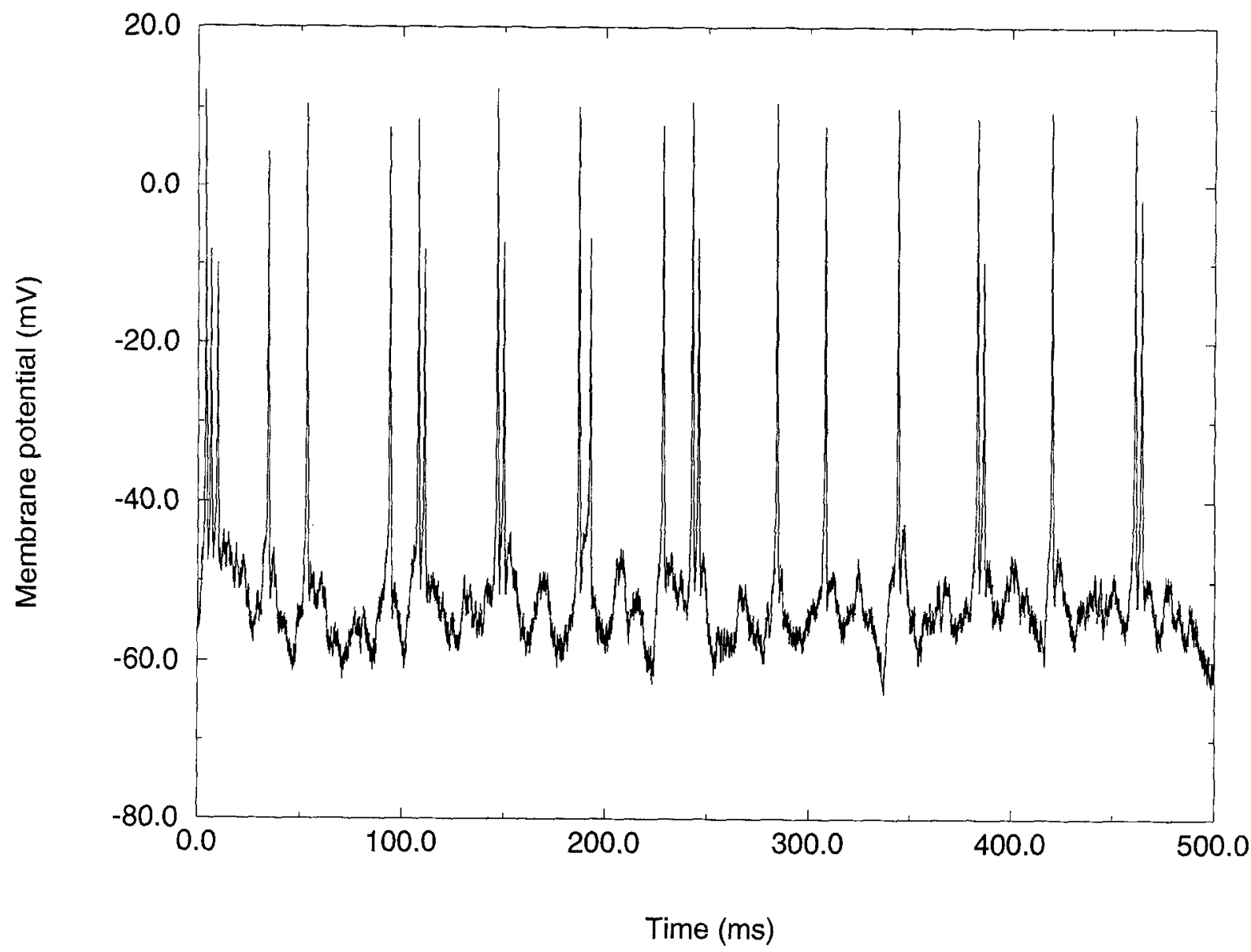

Figure 5. Higher resolution plot of the membrane potential of the 4th pyramidal cell from Fig. 4. There are a series of rhythmic compound EPSPs, some of which have spikes arising from them. Spikes do not occur at other times (in the 'troughs') because the membrane resistance is substantially reduced by a combination of inhibitory feedback and intrinsic potassium currents.

is important for producing synchronized population oscillations, but the strength of this inhibition should not be as great as that between inhibitory cells and their pyramidal targets.

\section{Synchronization between Columns}

Since synchronization has been observed between different cortical columns and even different cortical areas (Eckhorn et al., 1988; Engel et al., 1991b; Gray et al., 1989; Koenig et al., 1995; Kreiter and Singer, 1992) we performed simulations to examine how synchronization arises between two columns, both internally synchronized. Two columns of 100 neurons each were simulated, each column connected as in Fig. 3. Connections between the columns were then added to see if synchronization between them could be established.
Long-range connections in cortex are mediated by pyramidal cell axons, and these axons make most of their synapses on other pyramidal cells, although at a significantly lower density than within their own column (Hirsch and Gilbert, 1991; Kisvarday and Eysel, 1992; Martin, 1988). Therefore, we started by connecting the pyramidal cells between the two columns with a density of $2.5 \%$ (each pyramidal cell received a synapse from 2 pyramidal cells in the other column). In this case and all other simulations using pyramidalpyramidal connections only, we were not able to obtain good synchronization between the two columns (not shown). The internal synchrony of each column appeared to be degraded and the only clear tendency we noted was for the columns to sometimes oscillate 180 degrees out-of-phase with each other. With some parameter choices this was a very strong effect, but in 

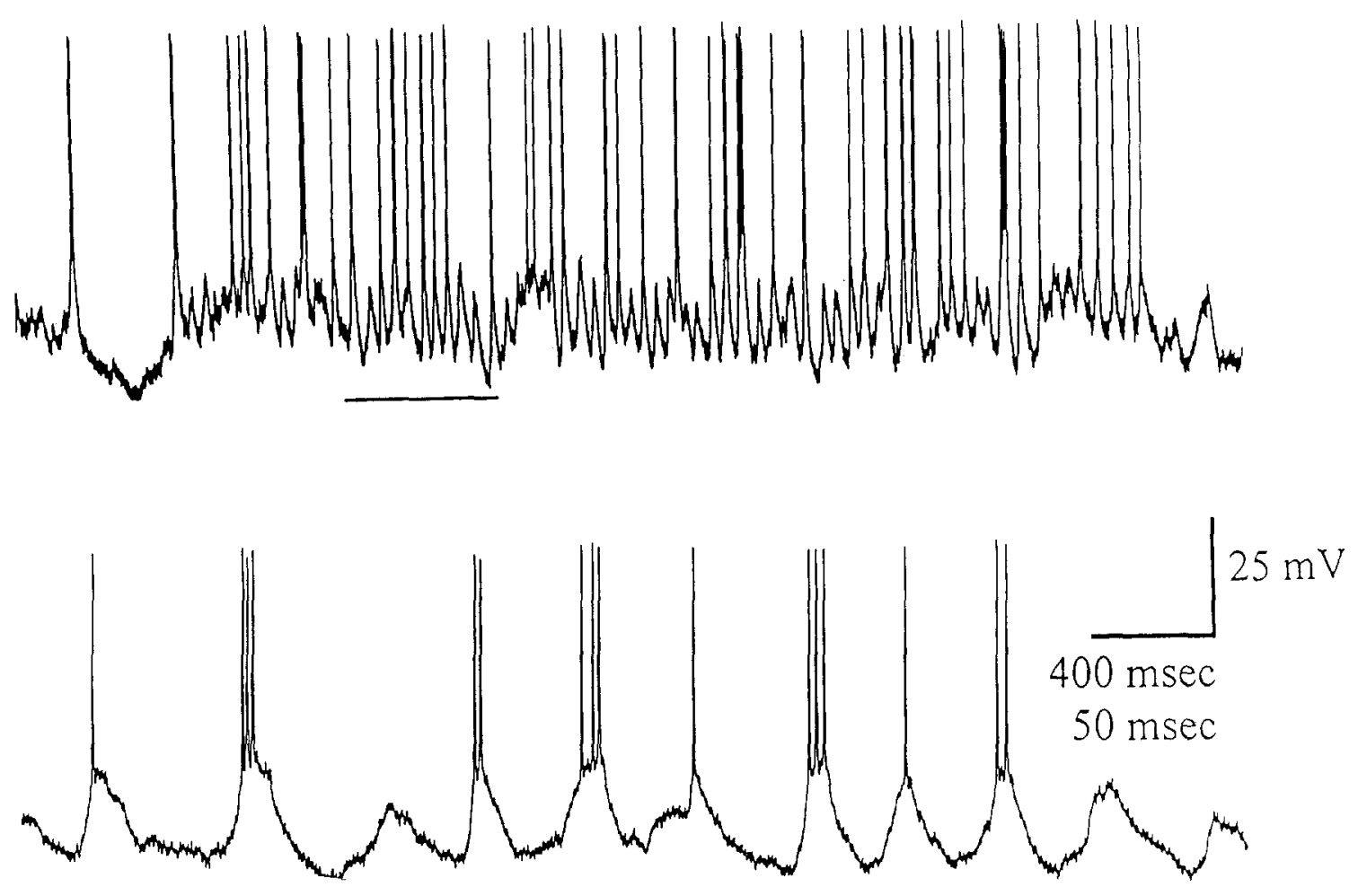

Figure 6. The response of a simple cell recorded intracellularly in area 17 of the cat to a square wave grating $(1 \mathrm{c} / \mathrm{deg})$ presented to the left eye at the optimal orientation and velocity. The data in the lower trace at higher time resolution was taken from the epoch in the upper trace that is marked by a horizontal line. The cell was tightly tuned for orientation and showed a monocular preference for the left eye. $V_{m}=-80 \mathrm{mV}, R_{\mathrm{in}}=57 \mathrm{M} \Omega$. This trace shows a similar series of compound EPSPs sometimes topped by spikes as the trace in Fig. 5. It is not known whether this neuron was an intrinsically bursting cell as assumed in the model. Cell recorded by CM Gray and DA MeCormick.

no case did clear synchronization with zero phase lag develop between the columns.

Long-range pyramidal cell axons make some of their synapses on dendritic shafts, many presumably belonging to inhibitory neurons (Douglas and Martin, 1990; White, 1989). Thus we added intercolumnar connections from pyramidal cells to basket cells to see if this would synchronize the two columns. Figure 8 shows the results of a simulation with two columns connected together at a density of $4 \%$ (each pyramidal cell received 3 synapses and each basket cell received 4 synapses from pyramidal cells in the other column). When the intercolumnar connections were turned on at $100 \mathrm{~ms}$, the two columns immediately hegan to synchronize $(\mathrm{CA}=0.61)$ and maintained near zero average phase difference $(1.2 \mathrm{~ms})$ for the duration of the simulation. When one column spontaneously desynchronized (e.g., at $350 \mathrm{~ms}$ ) the other did too, then both rapidly resynchronized. Intercolumnar synchronization could not be obtained by simply increasing the number of pyramidal-pyramidal connections. Thus the pyramidal-basket intercolumnar connection, while numerically small, was vital for intercolumnar synchronization.

Figure 9A shows the cross correlation of two LAPs from the simulation shown in Fig. 8. The central peak is at $-2.6 \mathrm{~ms}$ indicating tight synchronization between the two populations. For comparison Fig. 9B shows the cross correlation between two LAPs from a simulation in which the two columns were not connected $(\mathrm{CA}=$ 0.17 ). In this case there was a peak at some random non-zero position indicating that the two populations were not synchronized with each other.

The simulation of the synchronization between the two columns in Fig. 8 was extended for $6.5 \mathrm{sec}$. Figure 10B shows the averaged power spectra of the two LAPs, with a clear peak at $44 \mathrm{~Hz}$, the frequency of the population oscillation. There may be a smaller 


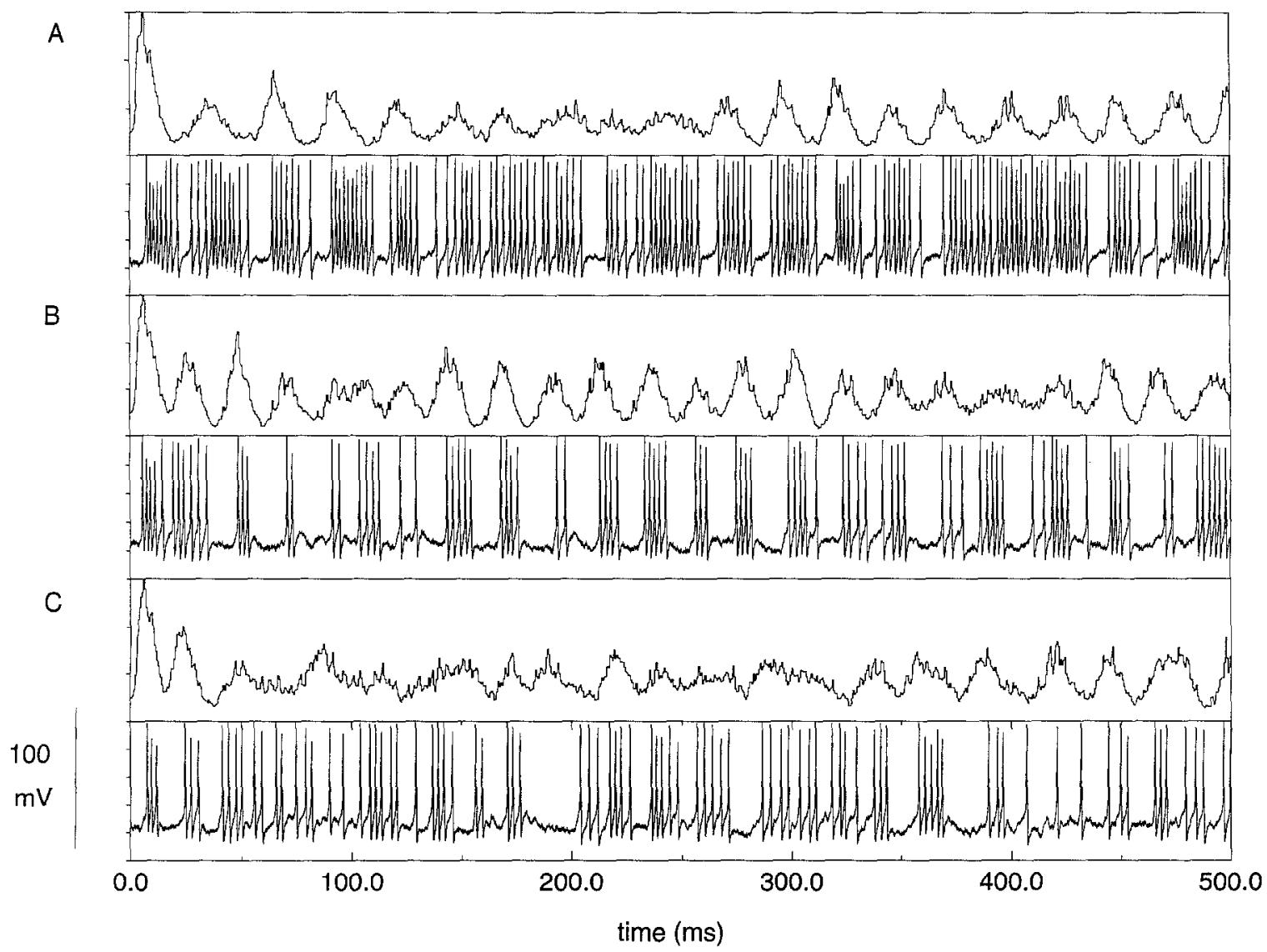

Figure 7. Effect of increasing the strength of reciprocal inhibition between the basket cells in a 100 neuron network. Inhibition increases from top to bottom. Each trace shows the population LAP and a sample basket cell trace. A) No inhibition between basket cells. Synchronization can still occur, but is not optimal because the discharges of the basket cells on each cycle perseverate, sometimes running into the next cycle. B) Basket cells connected by $1 \mathrm{nS}$ synapses. These traces are taken from Fig. 3. This is the optimal amount of mutual inhibition between basket cells in our model column. C) Basket cells connected by $2 \mathrm{nS}$ synapses. Synchronization is degraded because the basket cells inhibit each other too strongly before they can fire a coherent burst and effectively terminate the burst of firing in the pyramidal cell population.

peak near $22 \mathrm{~Hz}$, a subharmonic of the main peak. Figure I0A shows the difference between the phase spectra of the two LAPs over the same frequency range as the power spectra. This phase difference has large, random fluctuations at all frequencies except around the frequency of the population oscillation. In this region the phase difference was consistently small, and at $44 \mathrm{~Hz}$ it was almost zero. This is evidence that the two populations were oscillating at $44 \mathrm{~Hz}$ in phase with each other.

Figure 10C, D shows the same results for the two unconnected columns. Although there was significant power in both spectra around $40 \mathrm{~Hz}$, a single peak was not as clear. The phase difference did not decrease around $40 \mathrm{~Hz}$, indicating that the two columns were oscillating at random phase with respect to each other.
Increasing synaptic time delays between neurons within a column did not disrupt synchronization. However, increasing the synaptic delay of the intercolumnar connections adversely affected the synchronization of the two columns. As the delay was increased to 3-4 ms a phase shift of a few ms devcloped betwecn the two populations. When the intercolumnar delay was greater than $5 \mathrm{~ms}$ synchronization was severely disrupted. Figure 11 shows two columns connected with a mean delay of $7.2 \mathrm{~ms}$. The synchronization of the two columns was weak $(\mathrm{CA}=0.39)$ because the internal synchrony of each column was weak and sporadic. We conclude that to maintain effective synchronization two columns must be connected with a delay of approximately $5 \mathrm{~ms}$ or less. 'The implications of this result will be considered in the Discussion. 


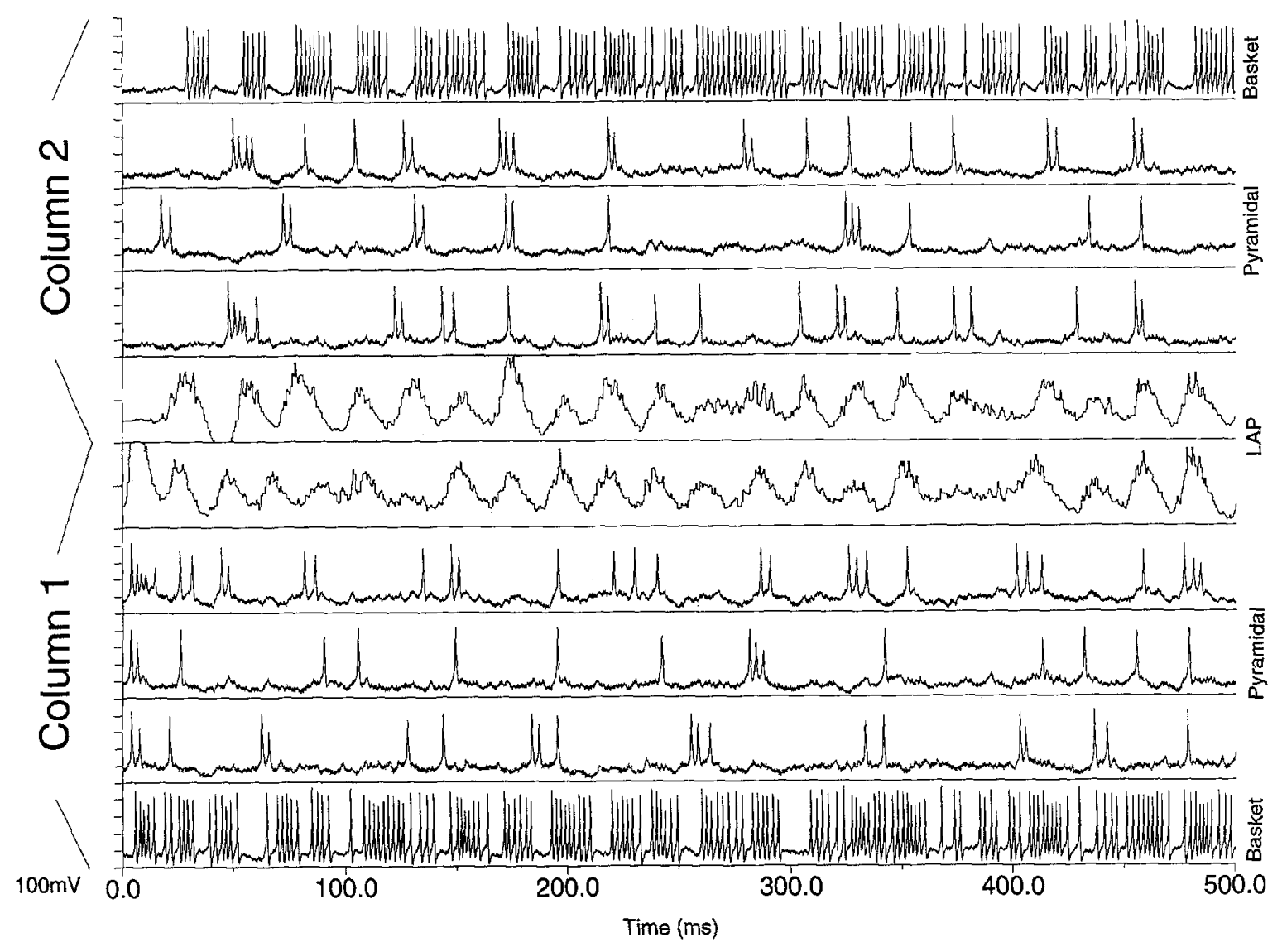

Figure 8. Zero phase lag synchrony between two columns of 100 neurons connected with pyramidal to pyramidal and basket cell synapses at a probability of $4 \%$. For each column a basket cell trace, 3 pyramidal cell traces and the LAP of all the pyramidal cells in that column are shown. Synchrony is rapidly established when the inter-columnar connections turn on at $100 \mathrm{~ms}$ and remains for the duration of the simulation. When the columns temporarily desynchronize and then resynchronize (e.g., at $375 \mathrm{~ms}$ ), they do so together.

\section{Effectiveness of Long-Range. Horizontal Axons}

The results of our model suggest that intercolumnar connections mediated by long-range horizontal axons are modulatory in function rather than directly excitatory, primarily because of inhibition directly evoked by long-range axonal stimulation. In order to test the validity of this result we have compared our model with an in vitro experiment directly testing the effects of long-range axonal stimulation. Figure 12 shows a simulation of an experiment performed in a cat cortical slice preparation (Fig. 7 of Hirsch and Gilbert (1991)). In the experiment, shocks of increasing strength were applied to lateral fibers in the upper layers, presumably stimulating horizontal pyramidal axons connecting distant columns. Synaptic responses were recorded from target pyramidal and presumed inhibitory interneurons. Our simulation produced the same results as the experiment: At low stimulus strengths EPSPs were observed in both pyramidal and basket cells. Due to the lower threshold of the basket cells, spikes were sometimes produced. As the stimulus strength increased the basket cells fired more spikes in response; in contrast, the pyramidal cells were inhibited by stronger shocks. This inhibition was a direct result of the response of the basket cells. Thus, due to the relatively low numbers of inter-columnar axons and the lower threshold of the target basket cells, the excitatory inter-columnar fibers do not necessarily have a strong excitatory effect on their target columns.

Figure 13 shows the output of two connected columns, the bottom one driven normally by external input, the top receiving no external input until $350 \mathrm{~ms}$. Although the top column received input from the bottom one that was sufficient to synchronize the two populations when they were both being driven (after 

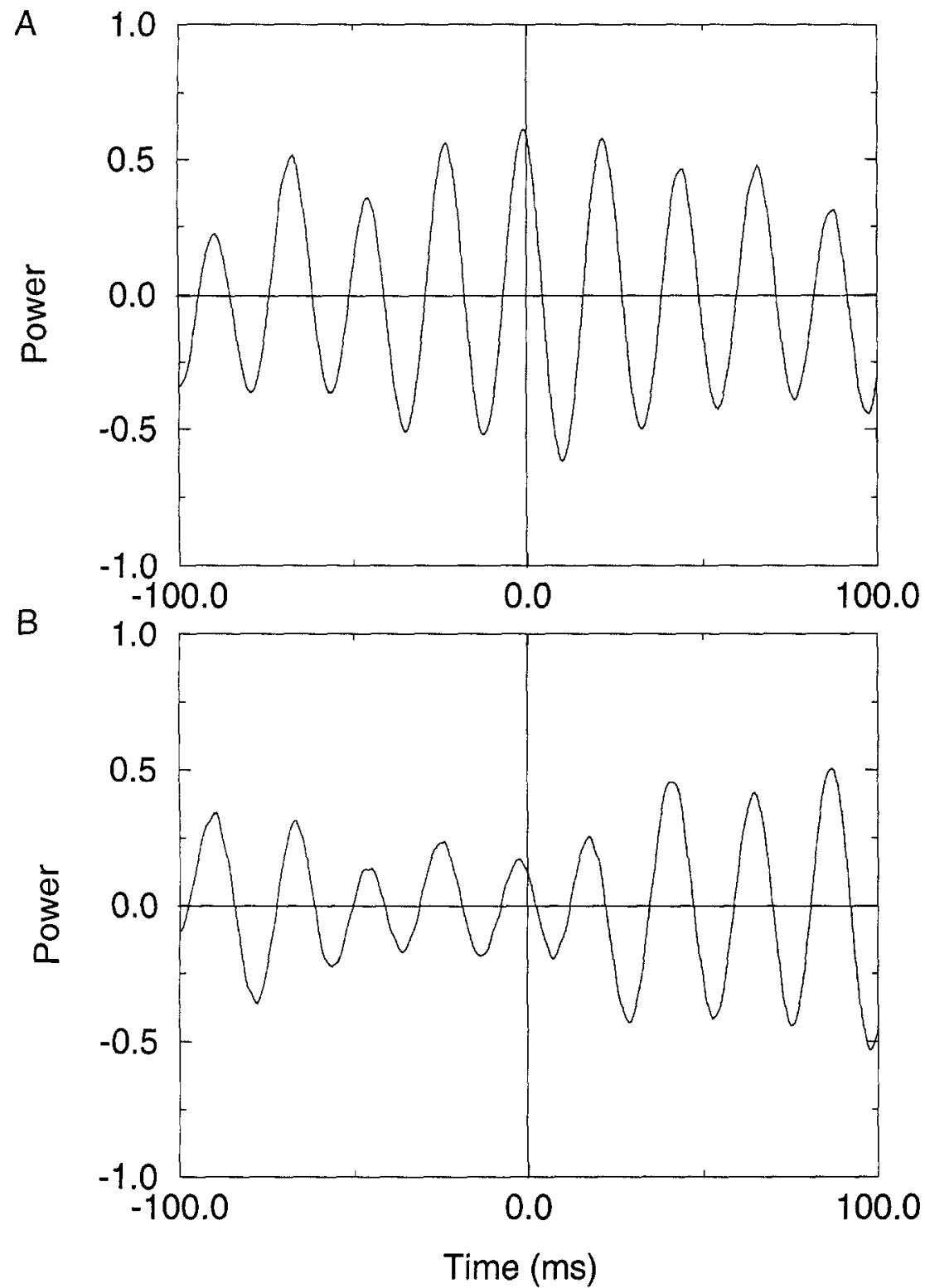

Figure 9. Cross-correlations of the LAPs of two-column simulations. A) Results from network shown in Fig. 8. There is a large central peak centered on $-2.6 \mathrm{~ms}$ which indicates that the two columns were oscillating in phase. B) Cross-correlation from two unconnected columns. The largest peak is at some random non-zero position indicating that these two columns were not oscillating in phase with each other.

$350 \mathrm{~ms}$ ), this input was not strong enough to cause significant firing before $350 \mathrm{~ms}$. The low firing rate in the top column was not significantly higher than the spontaneous rate due to noise.

\section{Discussion}

Synchronized oscillations have been demonstrated in a wide variety of models of interacting neurons ranging from models based on coupled intrinsic oscillators (Schuster and Wagner, 1990; Sompolinsky et al., 1990; Winfree, 1967; Williams, 1992) to more realistic models incorporating the characteristics of real neurons (Bush and Douglas, 1991; Sporns et al., 1989; Wilson and Cowan, 1972; Wilson and Bower, 1991). The former allow analysis while the latter permit detailed comparisons with recordings from cortical neurons. In particular, our realistic model was highly constrained 

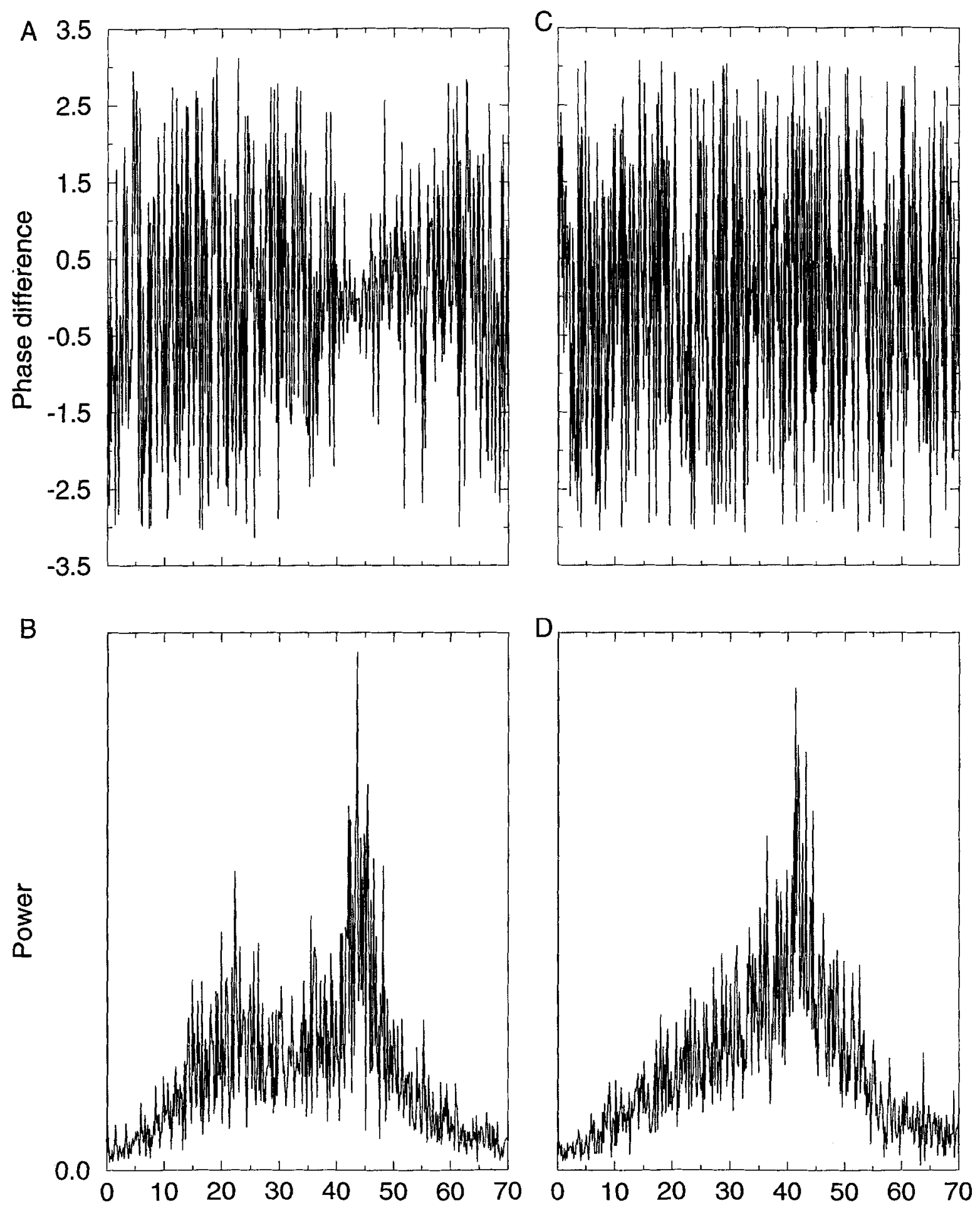

\section{Frequency $(\mathrm{Hz})$}

Figure 10. Phase difference between LAPs from two-column simulations. A) Difference between the phase spectra of the LAPs over the same frequency range as in (B). The phase difference decreases to zero at $44 \mathrm{~Hz}$, the oscillation frequency of the network, indicating zero-lag synchronization of the two columns. B) Averaged power spectra of the two LAPs of Fig. 8 (simulation continued for 6.5 secs). There is a large peak at $44 \mathrm{~Hz}$. C) Phase difference between two LAPs from two unconnected columns. There is no decrease in the phase difference around the oscillation frequency of the two columns confirming that they are not synchronized together. D) Averaged power spectra of the two LAPs. 


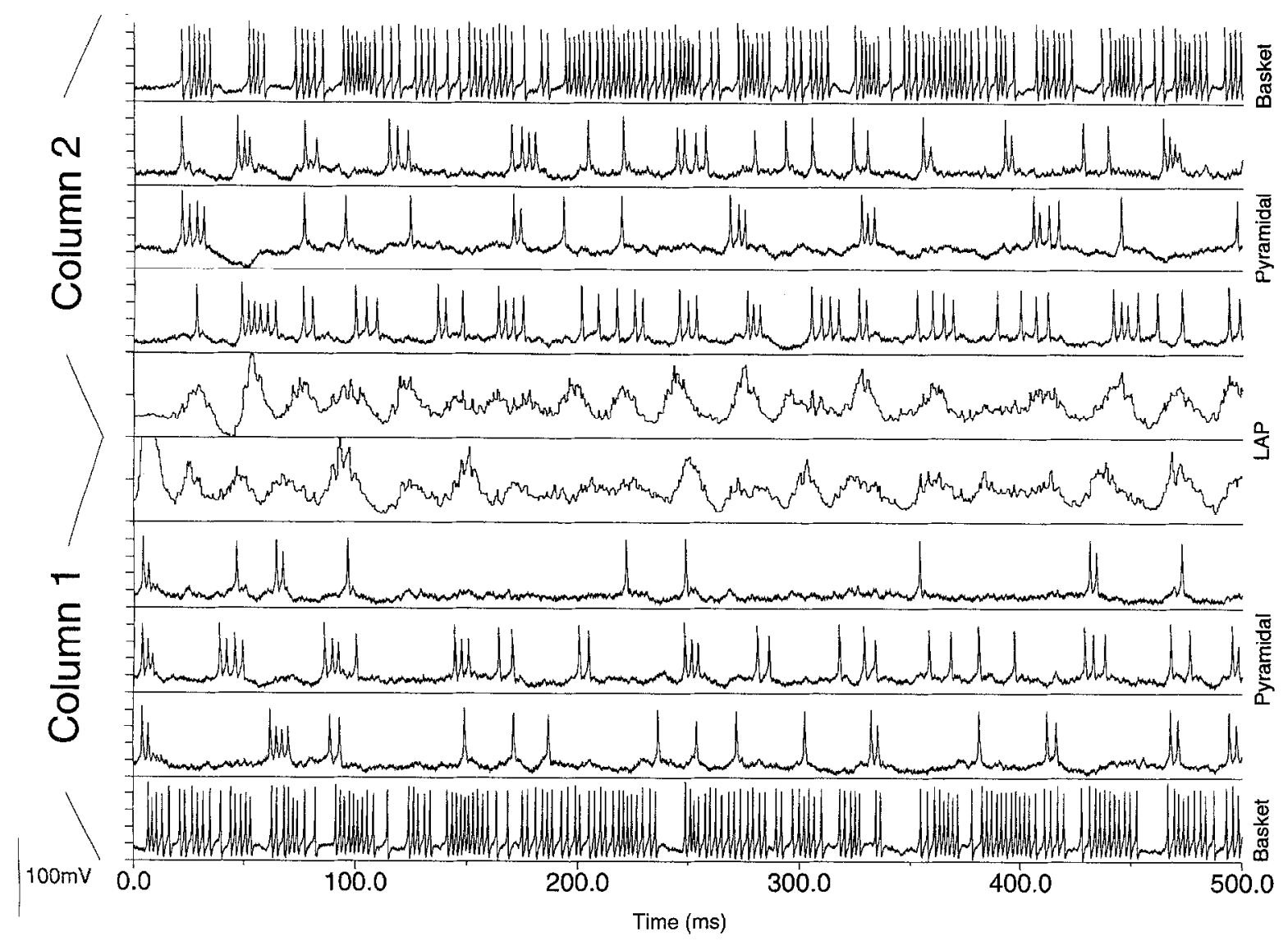

Figure 11. Synchrony between columns is severely degraded by increasing the inter-columnar time delay. These two columns were connected by synapses that had a mean delay of $7.2 \mathrm{~ms}$ and turned on at $100 \mathrm{~ms}$. Not only was the synchrony between the columns degraded by long time delays, but the internal synchrony within each column was also disrupted.

by the morphology and physiology of cortical neurons and the patterns of connectivity observed in primary sensory cortices. Parameters that were not fully constrained were varied over a wide range to find values that led to a match with physiological recordings. Our main results concern the essential role of inhibitory neurons in synchronizing collective oscillations within and between sparsely connected columns of cortical neurons.

\section{Synchronization in a Single Column}

The mechanism of synchronization is similar to that described previously (Bush and Douglas, 1991): Pyramidal cell burst discharges rapidly excite other pyramidal cells, producing a large compound EPSP in all cells in the network, including the inhibitory basket cells; the basket cells are driven to fire simultaneously, their feedback inhibition onto the pyramidal cells then terminates the population burst and, together with intrinsic potassium conductances, produces a post-burst hyperpolarization. Since the input resistance of the pyramidal cells is greatly reduced during this hyperpolarization (Bush and Sejnowski, 1994), there is less chance of the cell spiking during this time (out of phase with the oscillation). Cells with intrinsic bursting frequencies that vary over an octave $(15-30 \mathrm{~Hz})$ can be made to synchronize together at one frequency (e.g., $45 \mathrm{~Hz}$ ) by this mechanism. Cells with lower intrinsic frequencies tend to 'miss' cycles of the oscillation rather than fire out of phase and they tend to fire single spikes instead of bursts.

This mechanism also produced synchronization in networks of regular firing pyranidal cells that do not fire in bursts (not shown). The population EPSPs were smaller and shorter in duration without bursting and of course the pyramidal cells fired less spikes; thus, 

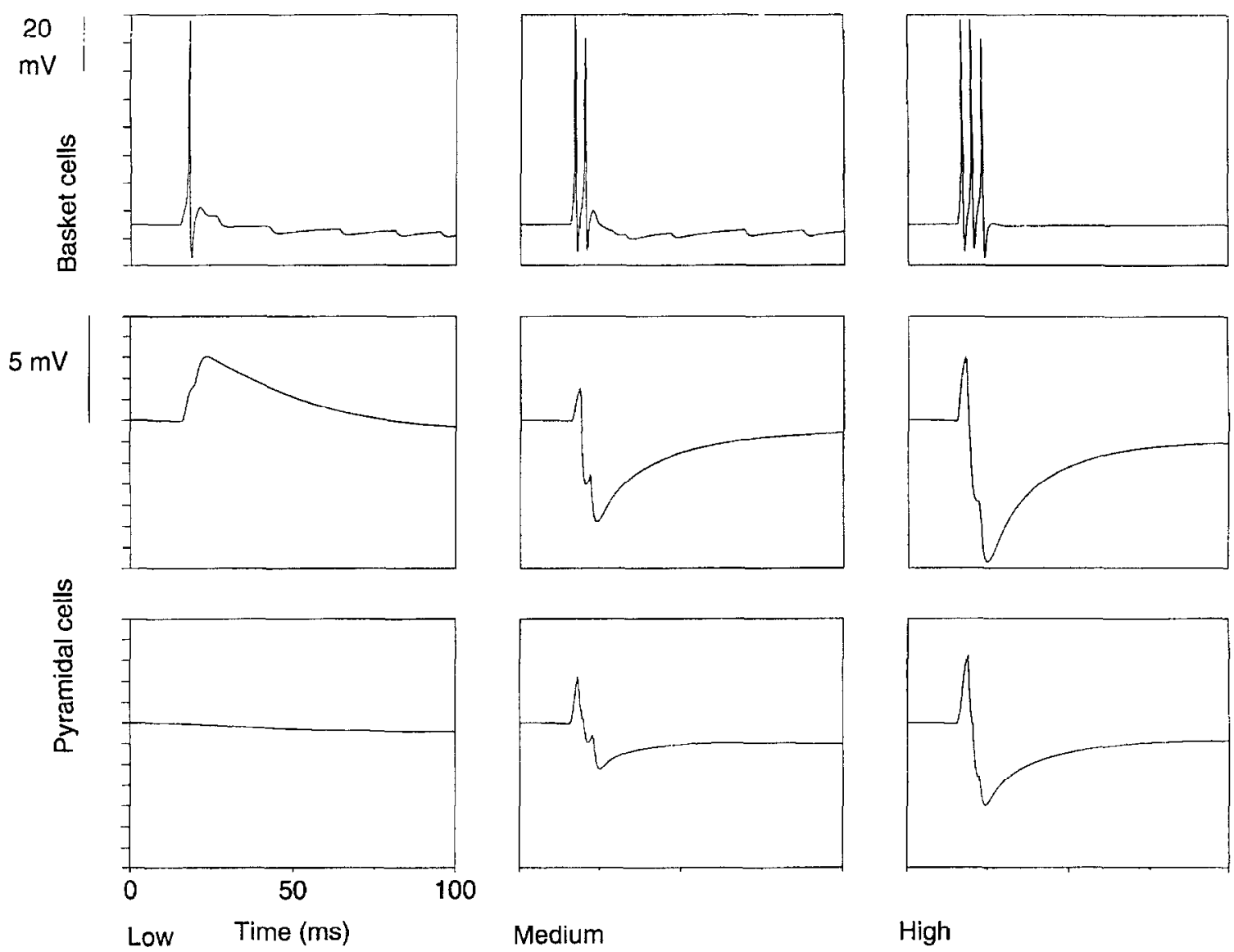

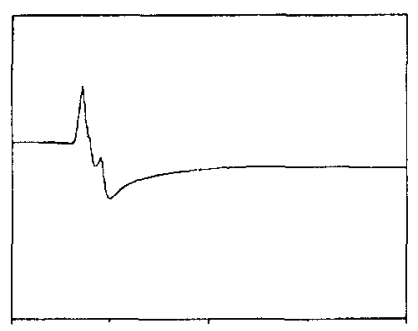

Medium

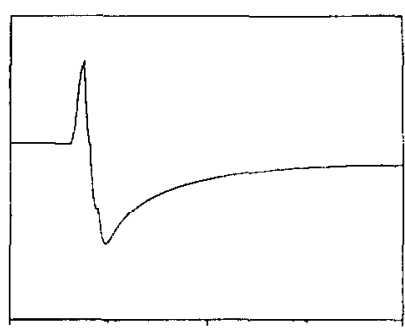

High

Figure 12. Response of a sample basket cell and two representative pyramidal cells to stimulation of inter-columnar connections. Increasing from left to right, 10,50 or 80 pyramidal cells in one column were stimulated with $0.5 \mathrm{nA}$ current for 8 ms. Postsynaptic responses were recorded in the other column. Synaptic scaling factor of intercolumnar connections was reduced from 10 to 3 to allow the simulation of stimulating a small number of fibers. Weak stimuli produce an EPSP in one of the pyramidal cells and a single spike in the basket cell. Stronger stimuli produce more spikes in the basket cells which cause IPSPs in the pyramidal cells, shutting off the excitatory response.

bursting improves but is not necessary for synchronization. A combination of bursting and regular firing cells also produced synchronization (not shown).

The synchronized oscillations demonstrated by the network shown in Fig. 3 display a number of features in common with experimental recordings (Engel et al., 1990): The similarity of the form of single cell traces from simulation and experiment (Figs. 5 and 6) suggests that the model has captured some of the most hasic characteristics of the biophysical mechanism of synchronous oscillatory firing in cortex. Intracellular recordings from cat striate cortex demonstrate oscillations that are stimulus dependent (absent during spontaneous activity) and increase in amplitude during stimulation while the cell is hyperpolarized (Bringuier et al., 1992; Jagadeesh et al., 1992). This suggests that the oscillations arose from rhythmic intracortical excitatory synaptic input. Of course other mechanisms may produce the same type of behavior (Llinas et al., 1991; McCormick et al., 1993), and several mechanisms may be involved. Recent experimental work in hippocampal slices has shown that, under certain conditions, $40 \mathrm{~Hz}$ oscillations may be produced by networks of inhibitory neurons driven by the activation of metabotropic glutamate receptors (Whittington et al., 1995).

Another feature observed in the network simulations was the variability of synchronization. In the model, as in the experimental data (Gray et al., 1992), periods of synchrony generally lasted a few hundred ms, with rapid spontaneous transitions into and out of the synchronized state. In addition, the presence of synchrony 


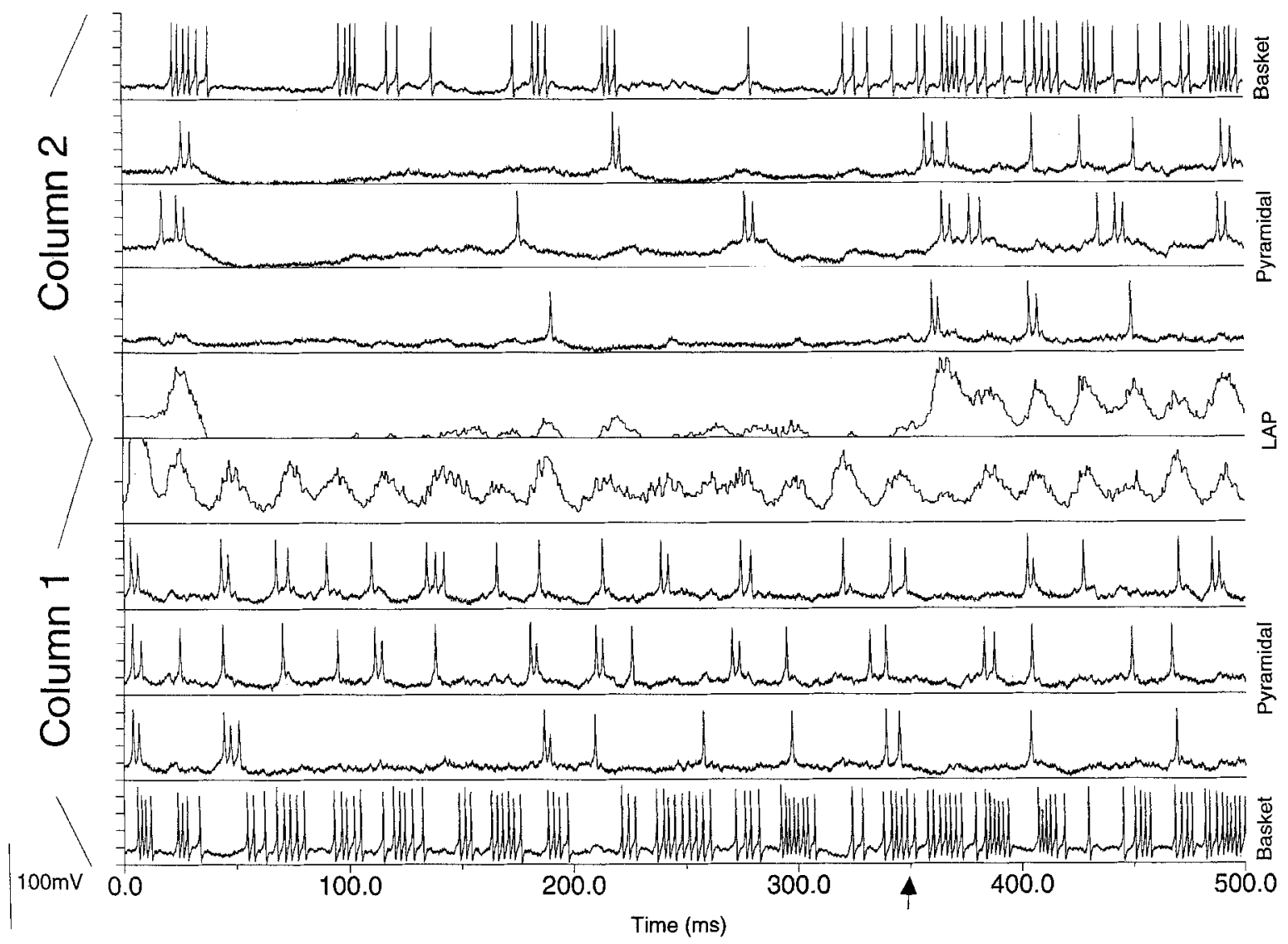

Figure 13. Inter-columnar connections have little effect on an undriven column. The two columns shown here are connected as in Fig. 8, with the inter-columnar connections turned on at zero $\mathrm{ms}$. The upper column receives no external driving input until $350 \mathrm{~ms}$ (arrow). After this time the two columns are synchronized by the inter-columnar connections, but before this they have very little effect on the undriven column, producing a few subthreshold depolarizations but few spikes. The basket cells have a lower threshold and fire a few spikes, which helps to suppress the pyramidal cells (see Fig. 12).

varied from trial to trial (using a different seed for the random number generator for noise and Poisson input). Throughout each trial the external driving input was a stationary Poisson process; thus, this variability cannot be due to changes in the statistics of the input and the complex internal dynamics of the network must be responsible for the rapid switching between synchronous and asynchronous firing. In the 1000 neuron simulation, however, the variability in the LAP was reduced (although individual cell traces still showed marked variability) (Fig. 4). Therefore, one possibility is that synchronized oscillations in neocortex are mediated by cell groups composed of about a hundred neurons. Synaptic strengths would have to be very large in a 100 neuron network (scaling factor of 10 , giving peak postsynaptic conductances of $10 \mathrm{nS}$ - see Methods). There is recent evidence that some single EPSPs could be this large, producing quantal depolarizations of a few $\mathrm{mV}$ instead of a few hundred $\mu \mathrm{V}$ (Thomson et al., 1993).

Networks with 1000 neurons used synaptic strengths without a scaling factor, suggesting that oscillating neuronal groups could comprise of on the order of 1000 neurons. This would fit better with a consideration of the number of cells involved: Given approximately 100,000 neurons $/ \mathrm{mm}^{3}$ and a column $100-200 \mathrm{~mm}$ in diameter there are on the order of 1000 neurons in one layer of one column. If this is the case, then the variability described above must be due to some source not included in the model. There are many potential candidates, including low probabilities of quantal release, use- and time-dependent potentiation and/or depression of synaptic strengths, the action of neuromodulators or some extrinsic cortical or subcortical signal. In a few 1000 neuron simulations, the probability of an EPSP/IPSP given a presynaptic action potential was 
reduced to 0.5 or lower (not shown): The amplitude and regularity of the LAP oscillation was significantly reduced. If this is not the case, and instead 100 neurons is the minimal size of an oscillating group, then a single layer in a single column could contain many such groups, which would seem unlikely in primary visual cortex, although association areas such as entorhinal cortex may support such smaller groups.

Experimental recordings also show a frequency variability of synchronized activity within and between trials ranging from $40-60 \mathrm{~Hz}$ (Gray et al., 1992). The model of Bush and Douglas (1991) displayed frequency variability within a single trial, but this was an artifact of having only one inhibitory neuron in their model: A single extra spike from this neuron would significantly delay the onset of the next population burst. We could obtain different frequencies of oscillation in our more realistic simulations only by changing the strength of the external driving input. In this way, the frequency could be changed in the range $35-60 \mathrm{~Hz}$. The external input was kept constant within a trial and a change in oscillation frequency was never observed. Therefore, we suggest that the frequency variability observed in experimental recordings is due to a concomitant variability in the strength of the extcrnal input. External here means external to the synchronized group, arising from the thalamus or other areas of cortex. For a pyramidal cell in one of our simulations, approximately half of its excitatory input was external stationary Poisson spike trains and half was excitatory feedback from other pyramidal cells in the network, the sole source of synchronized inputs.

There was no external input onto the basket cells in our model. If we included such input network synchrony was degraded. Thus, although external inputs to a cortical column do contact inhibitory cells as well as pyramidal cells (Douglas and Martin, 1990), our results suggest that the vast majority of input to the inhibitory interneurons in a synchronized oscillating cell group comes from excitatory (pyramidal) cells in that same group. Alternatively strong external inputs to inhibitory cells may be correlated, either from the thalamus or other cortical regions, as long as they are synchronized with that column.

\section{Synchronization between Columns}

Zero-phase lag synchrony between two populations was established within one or two cycles following activation of the intercolumnar connections (Figs. 8 and
13). Often the oscillation in one column would continue unperturbed while a cycle in the other would be suppressed or a new one prematurely initiated to get the two columns in phase. Connections from the pyramidal cells in a column to both the pyramidal cells and the basket cells in the other column were required. The greater effectiveness of inhibitory input in producing synchronization (Lytton and Sejnowski, 1991) may explain the need for pyramidal-basket intercolumnar connections. Reciprocal connections were not strictly necessary: Two columns could be synchronized with uni-directional connections from one to the other (not shown), although the synchrony obtained with this connectivity was not as strong as with reciprocal connections between the columns.

One parameter that did have a large effect on synchrony between columns was the delay time (Fig. 11). Our model predicts that two cortical areas cannot be synchronized by direct connections if the delay in those connections is significantly greater than $5 \mathrm{~ms}$ ( $\sim 1 / 4$ period), in agreement with previous models based on coupled oscillators (Schuster and Wagner, 1989; Sompolinsky et al., 1990). Interestingly, in the cat this does not appear to exclude any areas of cortex directly connected to each other, even if they are in opposite hemispheres. Time delays between cells in the same column, separated by tens of $\mu \mathrm{m}$, are 1-2 ms due to conduction along thin, unmyelinated axons (Mason et al., 1991; Thomson et al., 1988). Time delays between cells in opposite hemispheres, separated by several $\mathrm{cm}$, have been recorded at or below 5 ms due to conduction along thick, myelinated axons (McCourt et al., 1990). It would appear that the axons are organized to put cortical cells functionally next to each other. Thus, cells coupled by synchronization throughout the extent of the visual system can work on the same task simultaneously.

However, the same is not true for the cortex of the rabbit, where callosal conduction times are tens of $\mathrm{ms}$ (Swadlow, 1991), too long for synchronization to work by direct connections. Thus, for this animal either there is some other mechanism of synchronization, or it does without synchronized $40 \mathrm{~Hz}$ activity of its two hemispheres.

Our proposed mechanism of long-range synchronization relies on direct connections between the synchronized columns. Therefore, a demonstration of long-range synchrony persisting between 2 areas after their direct connections have been cut would suggest that instead synchrony is maintained through other 
pathways, for example common input from some subcortical source.

The results shown in Figs. 12 and 13 are consistent with the hypothesis that the horizontal connections in neocortex serve a modulatory role; in this case they serve to synchronize cortical columns. The strong effect of surround stimulation on a neuron that is also receiving direct stimulation of its receptive field (Gilbert and Wiesel, 1990) is also consistent with a modulatory role for the long-range horizontal connections. It should be noted that recent work has suggested that these connections can be strengthened under some conditions (perhaps by sprouting of extra axon collaterals) to the point where they can indeed drive cells in the $a b-$ sence of other input (Darian-Smith and Gilbert, 1994; Pettet and Gilbert, 1992).

\section{Conclusion}

Synchronization in model cortical networks is a robust phenomenon resistant to variation in parameters that are known to show a wide degree of variation in cortex. As long as time delays are short enough, which in the cat seems to hold even across hemispheres, cells in any number of directly connected cortical regions could firc in synchrony with each other regardless of where they are located physically. Our model is incomplete in some respects, but the central role of inhibition in promoting synchronous activity is likely to be robust; indeed, inhibition has been shown to be crucial for synchronization in other related models (Kopell and LeMasson, 1994; van Vreeswijk et al., 1994).

\section{References}

Bernander O, Douglas RJ, Martin KAC, Koch C (1991) Synaptic background activity influences spatiotemporal integration in single pyramidal cells. Proc. Nat. Acad. Sci. 88:11569-11573.

Borg-Graham LJ (1987) Modeling the Somatic Electrical Response of Hippocampal Pyramidal Neurons. M.S., MIT.

Bringuier V, Fregnac Y, Debanne D, Shulz D, Baranyi A (1992) Synaptic origin of rhythmic visually evoked activity in kitten area 17 neurones. Neuroreport 3(12):1065-1068.

Bush PC, Douglas RJ (1991) Synchronization of bursting action potential discharge in a model network of neocortical neurons. Neural Comp. 3:19-30.

Bush PC, Sejnowski TJ (1993) Reduced compartmental models of neocortical pyramidal cells. J. Neurosci. Methods 46:159-166.

Bush PC, Sejıowski TJ (1994) Effects of inhibition and dendritic saturation in simulated neocortical pyramidal cells. J. Neurophysiol. 71(6):2183-2193.
Connors BW, Malenka RC, Silva LR (1988) Two inhihitory postsynaptic potentials, and GABAa and GABAb receptor-mediated responses in neocortex of rat and cat. J. Physiol. 406:443-468.

Crick F, Koch C (1990) Towards a neurobiological theory of consciousness. Sem. Neurosci. 2:263-275.

Darian-Smith C, Gilbert CD (1994) Axonal sprouting accompanies functional reorganization in adult cat striate cortex. Nature 368:737-740

Douglas RJ, Martin KAC (1990) Neocortex. In: G Shepherd, ed. Synaptic Organization of the Brain. Oxford University Press, New York, pp. 220-248.

Eckhorn R, Bauer R, Jordan W, Brosch M, Kruse W (1988) Coherent oscillations: A mechanism for feature linking in the visual cortex. Biol. Cybern. 60:121-130.

Engel AK, Konig P, Gray CM, Singer W (1990) Stimulus-dependent neuronal oscillations in cat visual cortex: Inter-columnar interaction as determined by cross-correlation analysis. Eur. J. Neurosci. 2:588-606.

Engel AK, Konig P, Singer W (1991a) Direct physiological evidence for scene segmentation by temporal coding. Proc. Natl. Acad. Sci. USA 88:9136-9140.

Engel AK, Konig P, Kreiter AK, Singer W (1991b) Interhemisphric synchronization of oscillatory neuronal responses in cat visual cortex. Science 252:11177-11179.

Gabbot PLA, Martin KAC, Whitteridge D (1987) Connections between pyramidal neurons in layer 5 of cat visual cortex (area 17). J. Comp. Neurol. 259:364-381.

Gilbert CD, Hirsch JA, Wiesel TN (1990) Lateral interactions in visual cortex. Cold Spring Harbor Symposia on Quantitative Biology 55:663-677.

Gilbert CD, Wiesel TN (1990) The influence of contextual stimuli on the orientation selectivity of cells in primary visual cortex of the cat. Vis. Res. 30(11):1689-1701.

Gray CM, Konig P, Engel AK, Singer W (1989) Oscillatory responses in cat visual cortex exhibit inter-columnar synchronization which reflects global stimulus properties. Nature 338:334-337.

Gray CM, Engel AK, Koenig P, Singer W (1992) Synchronization of oscillatory neuronal responses in cat striate cortex: Temporal properties. Vis. Neurosci. 8:337-347.

Hines ML (1989) A program for simulation of nerve equations with branching geometries. Int. J. Biomed. Comp. 24:55-68.

Hirsch JA, Gilbert CD (1991) Synaptic physiology of horizontal connections in the cat's visual cortex. J. Neurosci. 11(6):18001809.

Jagadecsh B, Gray CM, Ferster D (1992) Visually evoked oscillations of membrane potential in cells of cat visual cortex. Science 257:552-554.

Kisvarday ZF, Eysel UT (1992) Cellular organization of reciprocal patchy networks in layer III of cat visual cortex (area 17). Neurosci. 46(2):275-286

Koch C, Douglas R, Wehmeier U (1990) Visibility of synaptically induced conductance changes: Theory and simulations of anatomically characterized cortical pyramidal cells. J. Neurosci. 10:17281744.

Koenig P, Schillen TB (1991) Stimulus-dependent assembly formation of oscillatory responses: I. synchronization. Neural Comp. 3:155-166.

Koenig P, Engel AK, Singer W (1995) Relation between oscillatory activity and long- range synchronization in cat visual cortex. Proc. Natl. Acad. Sci. USA 92:290-294. 
Komatsu Y, Nakajima S, Toyama K, Fetz EE (1988) Intracortical connectivity revealed by spike-triggered averaging in slice preparations of cat visual cortex. Brain Res. 442:359-362.

Kopell N, LeMasson G (1994) Rhythnogenesis, amplitude modulation, and multiplexing in a cortical architecture. Proc. Natl. Acad. Sci. USA 91:10586-10590.

Kreiter AK, Singer W (1992) Oscillatory neuronal responses in the visual cortex of the awake macaque monkey. Eur. J. Neurosci. 4:369-375.

Llinas RR, Grace AA, Yarom Y (1991) In vitro neurons in mammalian cortical layer 4 exhibit intrinsic oscillatory activity in the $10-$ to $50-\mathrm{Hz}$ frequency range. Proc. Natl. Acad. Sci. USA, 88(3):897-901.

Lytton WW, Sejnowski TJ (1991) Simulations of cortical pyramidal neurons synchronized by inhibitory interneurons. J. Neurophysiol. 66:1059-1079.

Martin KAC (1988) From single cells to simple circuits in the cerebral cortex. Q. J. Exp. Physiol. 73:637-702.

Mason A, Nicoll A, Stratford K (1991) Synaptic transmission between individual pyramidal neurons of the rat visual cortex in vitro. J. Neurosci. 11(1):72-84.

McCormick DA, Gray C, Wang Z (1993) Chattering cells: A new physiological subtype which may contribute to 20 $60 \mathrm{~Hz}$ oscillations in cat visual cortex. Soc. Neurosci. Abst. 19:359.9.

McCourt ME, Thalluri G, Henry GH (1990) Properties of area 17/18 border neurons contributing to the visual transcallosal pathway in the cat. Vis. Neurosci. 5(1):83-98.

Nicoll A, Blakemore C (1990) Dual intracellular impalement in vitro reveals excitatory synaptic connections between pyramidal neurons in rat visual cortex. $J$. Physiol. p. 15.

Nicoll A, Rlakemore C (1993) Patterns of local connectivity in the neocortex. Neural Comp. 5:665-680.

Pettet MW, Gilbert CD (1992) Dynamic changes in receptive-field size in cat primary visual cortex. Proc. Natl. Acad. Sci. USA 89:8366-8370.

Rall W (1964) Theoretical significance of dendritic trees for neuronal input-output relations. In: R Reiss, ed., Neural Theory and Modeling (pp. 73-97). Stanford: Stanford University Press.

Schuster HG, Wagner P (1989) Mutual entrainment of two limit cycle oscillators with time delayed coupling. Prog. Theor. Phys. 81(5):939-945.

Schuster HG, Wagner P (1990) A model for neuronal oscillations in the visual cortex. 1. Mean-field theory and derivation of the phase equations. Biol. Cybernet. 64(1):77 82 .
Silva LR, Amitai Y, Connors BW (1991) Intrinsic oscillations of neocortex generated by layer 5 pyramidal neurons. Science 251:432435.

Singer W (1993) Synchronization of cortical activity and its putative role in information processing and learning. Ann. Rev. Physiol. 55:349-374.

Somogyi P, Kisvardy ZF, Martin KAC, Whitteridge D (1983) Synaptic connections of morphologically identified and physiologically characterized large basket cells in the striate cortex of cat. $\mathrm{NeU}$ rosci. 10(2):261-294.

Sompolinsky H, Golomb D, Kleinfeld D (1990) Global processing of visual stimuli in a neural network of coupled oscillators. Proc. Natl. Acad. Sci. USA 87(18):7200-7204.

Sporns O, Gally JA, Reeke GN, Edelman GM (1989) Reentrant signalling among simulated neuronal groups leds to coherency in their oscillatory activity. Proc. Natl. Acad. Sci. USA 86:7265-7269.

Sporns O, Tononi G, Edelman GM (1991) Modeling perceptual grouping and figure- ground separation by means of active reentrant connections. Proc. Natl. Acad. Sci. USA 88:129-133.

Swadlow HA (1991) Efferent neurons and suspected interneurons in second somatosensory cortex of the awake rabbit: Receptive fields and axonal properties. J. Neurophysiol. 66(4):1392-1408.

Thomson AM, Girdlestone D, West DC (1988) Voltage-dependent currents prolong single-axon postsynaptic potentials in layer III pyramidal neurons in rat neocortical slices. $J$. Neurophysiol. 60:1896-1907.

Thomson AM, Deuchars J, West DC (1993) Largc, decp laycr pyramid-pyramid single axon EPSPs in slices of rat motor cortex display paired pulse and frequency-dependent depression, mediated presynaptically and self-facilitation, mediated postsynaptically. J. Neurophysiol. 70(6):2354-2369.

van Vreeswijk C, Abbott LF, Ermentrout GB (1994) When inhibition not excitation synchronizes neural firing. J. Comp. Neurosci. 1(4).

White EL (1989) Cortical Circuits. Birkhauser, Boston.

Whittington MA, Traub RD, Jefferys JGR (1995) Synchronized oscillations in interneuron networks driven by metabotropic glutamate receptor activation. Nature 373:612-615.

Williams TL (1992) Phase coupling by synaptic spread in chains of coupled neuronal oscillators. Science 258:662-665.

Wilson HR, Cowan JD (1972) Excilatory and inhibitory interactions in localized populations of model neurons. Biophys. J. 12:1-24.

Wilson WA, Bower JA (1991) A computer simulation of oscillatory behavior in primary visual cortex. Neural Comp. 3:498-509.

Winfree AT (1967) Biological rhythms and the behavior of populations of coupled oscillators. J. Theor. Biol. 16:15-42. 